\title{
THE SENSITIVITY OF INVESTMENT CASTING SIMULATIONS TO THE ACCURACY OF THERMOPHYSICAL PROPERTY VALUES
}

\author{
X. L. Yang ${ }^{1}$, P. D. Lee ${ }^{1}$, R. F. Brooks ${ }^{2}$, R.Wunderlich ${ }^{3}$ \\ ${ }^{1}$ Material Processing Group, Department of Materials, Imperial College London \\ Prince Consort Road, SW7 2BP, London, UK \\ ${ }^{2}$ NPL Materials Centre, Teddington, TW11 2UL, UK \\ ${ }^{3}$ Universität Ulm, Albert-Einstein-Allee 47, D89081 Ulm, Germany
}

Keywords: Measurements, Thermophysical properties, Simulation, Sensitivity, Directional solidification

\begin{abstract}
In this study, the importance of the accuracy of the thermophysical property to a simulation of an investment cast directionally solidified nickel-based superalloy turbine blade was investigated. CMSX-4 was chosen as the base alloy. The effect of the thermophysical properties on the thermal field and solidification microstructure was studied using a coupled macromesoscale model. Relevant thermophysical properties examined include density, specific heat (enthalpy) and thermal conductivity in both the liquid and the solid phases.

Commercial finite-element analysis software, ProCAST, was used as the macroscale model to solve the heat and mass transportation equations. An in-house mesoscale code was used to simulate the dendritic solidification structure including microsegregation. During the macroscale analysis, the simulation results were compared to an idealized turbine blade previously cast under industrial conditions. In the mesoscale simulation, the response of primary arm spacing and segregation range was examined. The temperature prediction and the thermal gradient in the liquid show the greatest sensitivity to density and solid state thermal conductivity. Primary arm spacing is most sensitive to density and specific heat. Segregation range shows greatest sensitivity to density and thermal diffusivity in the solid.
\end{abstract}

\section{Introduction}

Computer based models are increasingly used to simulate industrial processes and are recognized as valuable tools to aid improved process control and product quality. One important application is the use of heat transfer and fluid flow models to design the molds used to produce cast metal components [1]. The conditions for filling the mold with metal, and its subsequent solidification, can be modeled to predict turbulence, feeding difficulties, shrinkage and porosity in the finished product. With this information the design of the mold or running system can be modified easily before going into production. The advent of inexpensive, powerful computing has allowed the models to become more complex, expanding their application beyond just macro defects to also predict the microstructural evolution. These models have now reached the stage where one of the limiting factors in their applicability is the accuracy of the thermophysical data for the materials to be modeled [1]. Accurate thermophysical data are difficult to obtain at high temperatures, especially for reactive alloys such as titanium and nickel-based superalloys [2]. Reliable thermophysical property data for the materials involved in the process is one of the prime requirements to improve the quality of the prediction.

As part of a project partly funded by the European Space Agency (ESA), a selection of thermophysical properties were measured for a series of alloys in several European laboratories as a precursor to performing measurements in a microgravity environment. These microgravity experiments were proposed to determine the effects of convection in the liquid samples, a factor which can be detrimental to the accurate measurement of certain properties, and is difficult to counteract under normal terrestrial conditions. From these initial terrestrial measurements, a set of recommended values were prepared and used in a sensitivity study as the base case and to indicate the uncertainties of measurement.

The aim of this study is to investigate the sensitivity of investment casting simulations to the accuracy of thermophysical properties. The measurement methods for relevant thermophysical properties are given followed by the description of the macro-meso scale model. The sensitivity of the macroscopic results to the thermophysical property change is then predicted followed by the response of the solidification microstructures.

\section{Measured Data}

Measurements on a single batch of CMSX-4 were made in a number of European laboratories. Heat capacity and enthalpy values were obtained by differential scanning calorimetry (DSC). This technique is widely used and is adequately described elsewhere [3]. Provided great care is taken, contamination of the samples can be minimized. However, results may be smeared due to the constant heating rate employed, and the response time of the instrument can cause error in the measurements. In the extended measurement study a variety of heating rates were used in order to minimize the errors. This method also provides values of liquidus and solidus, although measurements taken from the cooling cycle may be affected by undercooling. The data can give an estimate of the fraction solid through the melting range, although these values assume the same specific heat for all phases evolved during solidification.

Density was measured by droplet imaging techniques, either by sessile drop, or by levitated drop methods, described elsewhere [4]. The data in this study agreed well for this alloy, but levitation methods, used because of their very high temperature capabilities, and low risk of sample contamination, suffer because of limitations in the accuracy of infra-red pyrometry under non-ideal 
conditions and because of asymmetry in the droplet shape caused by oscillations in the drop shape.

Thermal conductivity is rarely measured directly in liquid metals as convection disrupts steady state methods, and reactions between sample and an immersed, metal based, probe prevent the use of most transient methods. Instead, measurements of thermal diffusivity are usually made using a laser flash method [5]. In the liquid, the sample must maintain a known geometry with parallel top and bottom surfaces. This requires the sample to be constrained in a cell, or container, and a correction made for that container when making a measurement. Reactions must also be avoided. No measurement is possible in the melting range of the alloy, as the heat pulse generated for the measurement will melt the structure of the sample rather than diffuse through it. As data for the interdendritic liquid compositions are rarely available, values are often taken for the liquid alloy at the liquidus, and solid alloy at the solidus, and applied assuming additivity throughout the melting range. Thermal conductivity is derived from the product of the thermal diffusivity, the heat capacity and the density, and so accurate measurements of all three properties are required.

\section{Model Description}

The thermal field was calculated by a macroscale code whilst solidification microstructure was simulated using an in-house mesoscale code. Details of the two models and the coupling method are given below.

\section{Macroscale Model}

A three-dimensional finite element solver, ProCAST (ESI Software, Paris) was applied to solve for the heat transfer during solidification at the macroscale. The model solves the heat transfer using the conservation of energy with temperature/time boundary conditions and temperature dependent material properties [6]:

$$
\rho \frac{\delta H}{\delta T} \frac{\delta T}{\delta t}-\nabla[K \nabla T]-S(r)=0
$$

where $\rho$ is density, $T$ is temperature, $t$ is time, $K$ is thermal conductivity, $S(r)$ is the source term and $H$ is the enthalpy of solidification. Substituting $S(r)$ with the latent heat of fusion during solidification process, Eq. (1) can be rewritten as:

$$
\rho C_{\mathrm{P}} \frac{\delta T}{\delta t}=\nabla[K \nabla T]+\rho L\left(1-f_{\mathrm{S}}(T)\right)
$$

where $C_{\mathrm{P}}$ is the isobaric specific heat, $L$ is the latent heat of fusion and $f_{\mathrm{S}}$ is the fraction solid.

A radiation boundary condition was considered in the macrosacle model. The radiation was calculated using a view factor model: rather than tracking the reflected radiant energy from each element, an overall energy balance for each participating surface is considered [7].

A schematic diagram of the model arrangement is shown in Fig. 1a. It contains the furnace chamber and casting elements. A cluster of four blades were cast together, allowing only one

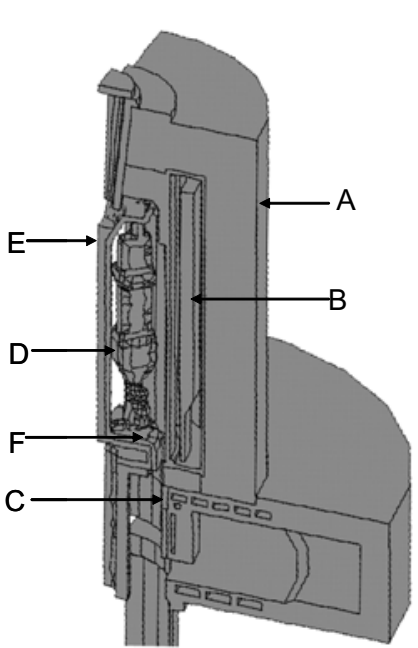

(a)

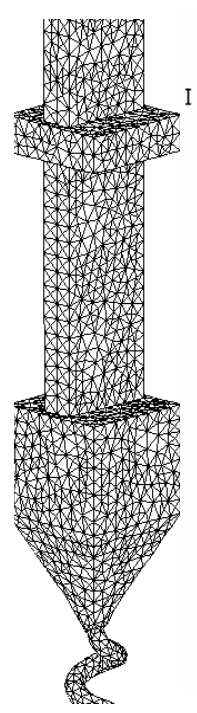

(b)

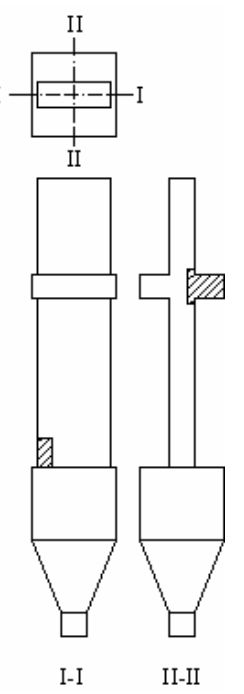

(c)
Fig. 1. Schematic drawing of the arrangement in macroscale model. (a) the whole assembly; (b) the finite element meshing showing a detail of idealized investment cast blade geometry; (c) locations of simulation domain in mesoscale calculations (indicated by hatched area). A: insulation wall; B: heating graphite; C: baffle; D: mold shell; E: casting; F: water cooled copper chill.

quarter of the furnace chamber to be considered due to symmetry. The meshing of the idealized casting blade is shown in Fig. $1 \mathrm{~b}$.

The shell mold is mounted on a water cooled copper chill in the center of the hot chamber. The mold is preheated to a temperature above the liquidus. After pouring of the molten metal the hot chamber is removed upwards rapidly until the starter and the selector were clear of the water cooled baffle; a withdrawal rate of $63.5 \mu \mathrm{m} / \mathrm{s}$ is maintained thereafter.

The calculated temperature in the model was compared to the measured temperature from a fully instrumented industrial directional casting blade (Rolls-Royce Company Research and Development Foundry, Bristol, UK). The temperature field in the vicinity of the selector, the blade body and shell mold has been examined previously $[8,9]$, illustrating a good correlation to the experimental results. Therefore, the thermal model has been assumed to be a valid tool for thermophysical data sensitivity study and as a basis for the microstructural model.

\section{Mesoscale Model}

The mesoscale model combines a description of grain growth by cellular-automaton (CA) with a finite-difference (FD) computation of solute diffusion. Both the $\mathrm{CA}$ and FD components of the model use the same square grid and time step. The model solves the solute conservation equation subjected to equilibrium conditions at the interface. The equilibrium data is obtained from a linearised phase diagram for a binary system. Details of the model have been published previously $[10,11]$, therefore only the key equations and assumptions are detailed below. 
The model starts with all cells in a liquid state, from which they may transform to a partly solid (growing) state by either nucleation or growth from a neighboring cell. In a growing cell, solute is partitioned between the solid and liquid phases. The change in solute concentration affects the degree of local undercooling and therefore affects the nucleation and growth processes.

In a growing cell, solute partitioning at the solid/liquid (S/L) interface is determined by:

$$
C_{\mathrm{S}}=k C_{\mathrm{L}}
$$

where $C_{\mathrm{S}}$ and $C_{\mathrm{L}}$ are the solute concentrations in solid and liquid phases, respectively. An equivalent concentration, $C_{\mathrm{E}}$, is defined for a growing cell as:

$$
C_{\mathrm{E}}=C_{\mathrm{L}}\left(1-f_{\mathrm{S}}\right)+C_{\mathrm{S}} f_{\mathrm{S}}=C_{\mathrm{L}}\left[1-(1-k) f_{\mathrm{S}}\right]
$$

where $f_{\mathrm{S}}$ is the solid fraction. At time $t$, the diffusion of solute in the liquid and solid is given by Fick's second law, and can be expressed in a general form:

$$
\frac{\partial C_{\mathrm{E}}}{\partial t}=\nabla \square\left(D_{\mathrm{E}} \nabla C_{\mathrm{E}}\right)
$$

where $D_{\mathrm{E}}$ is an equivalent diffusion coefficient, defined as:

$$
D_{\mathrm{E}}=\left(1-f_{\mathrm{S}}\right) D_{\mathrm{L}}+f_{\mathrm{S}} k D_{\mathrm{S}}
$$

where $D_{\mathrm{L}}$ and $D_{\mathrm{S}}$ are diffusion coefficients in liquid and solid, respectively. Substituting the equivalent diffusion into Eq. (5), one can get:

$$
C_{\mathrm{L}}(1-k) \frac{\partial f_{\mathrm{S}}}{\partial t}=-\nabla \llbracket\left(D_{\mathrm{E}} \Delta C_{\mathrm{L}}\right)+\left[1-(1-k) f_{\mathrm{S}}\right] \frac{\partial C_{\mathrm{L}}}{\partial t}
$$

Eq. (7) is the governing equation for obtaining the solid fraction change, $d f_{\mathrm{S}}$, in the growing cells, and is solved using an explicit FD scheme. The calculated fraction solid change is used to correlate the growth rate in a growing cell according to the decentred algorithm [11].

The solid growth is controlled by both the diffusion limited solute built up and the crystallographic anisotropy simulated by a modified decentred square growth algorithm based on the work of Gandin et al. [12]. The S/L interface velocity is first estimated using the local (assuming local equilibrium) undercooling from the prior time step. Details of the growth model are explained elsewhere [11].

The mesoscale model has previously been validated against experimental results reported elsewhere [13].

\section{Coupling the Macro-meso Scale Model}

To link the macroscale model with the mesoscale model, the spatial domain must be superimposed. Given a finite element mesh, a CA mesh is generated in order to correspond to each element. The CA grid can be applied locally for a given region of the FE mesh. Once the CA grid is generated, shape functions are used to interpolate the values from the FE nodes. Therefore temperature can be obtained for each of the cells in the CA grid.

In addition to the spatial domain, the temporal field is also linked. The typical macro timestep used in the FE solver is 1 to $5 \mathrm{~s}$ which is several orders of magnitude greater than a typical timestep used in the CA (several ms). For each macro step many meso steps are calculated.

At present, the two models are weakly-coupled: at each macro time step the temperatures are interpolated to the cell locations in the mesoscale model. However, there is no feedback of latent heat released at the mesoscale model: latent heat is taken into account at the macroscale level during the calculation in ProCAST.

\section{Macroscale Model Sensitivity}

The sensitivity of the thermal field to the variation in thermophysical property values is investigated in this section. The variation studied for each thermophysical property is listed in Table 1 . The values in the second column are the ranges indicative of the variations found with experimental measurements. In addition, an extended range (column 3) was also examined for each of the properties to gain a better understanding of the sensitivity of the model responses to that parameter. Thermophysical property data from Mills [14] for CMSX-4 was used in this study as the base values. The temperature distribution, thermal gradient in the liquid at $\mathrm{S} / \mathrm{L}$ interface $\left(G_{\mathrm{L}}\right)$, and local solidification time $\left(t_{\mathrm{s}}\right)$ were examined.

Table 1. Variation range of thermophysical property.

\begin{tabular}{ccc}
\hline Thermophysical property & Range 1 (\%) & Range 2 (\%) \\
\hline Density & \pm 2 & \pm 10 \\
Specific heat & \pm 5 & \pm 15 \\
Thermal conductivity in liquid & \pm 10 & \pm 15 \\
Thermal conductivity in solid & \pm 4 & \pm 15 \\
\hline
\end{tabular}

\section{Temperature Distribution}

In Fig. 2, the influence of changing thermophysical properties on the temperature distribution is illustrated by comparison with the base case at the time when it reaches two separate stages of solidification at the thermocouple located in the platform region. The two stages are: 1) the start of solidification (liquidus temperature); and 2) a fraction solid of 0.5. These two temperatures are thought to bound the range of temperatures where freckles initiate.

Fig. 2a shows the temperature variation (the temperature difference between changed thermophysical properties and the base case) at the liquidus temperature while Fig. $2 \mathrm{~b}$ shows the variation at 0.5 fraction solid. The temperature variation is relatively small (within $\pm 4{ }^{\circ} \mathrm{C}$ ) at the liquidus temperature, increasing with fraction solid. At a fraction solid of 0.5 , the temperature variation is between -12 and $8{ }^{\circ} \mathrm{C}$.

Positive variation in density increases the predicted temperature difference, whereas a negative variation decreases it. The temperature variation also increases with fraction solid: with $5^{\circ} \mathrm{C}$ difference at liquidus temperature and $8{ }^{\circ} \mathrm{C}$ at 0.5 fraction solid when density is increased by $10 \%$. This is due to the effect of the 


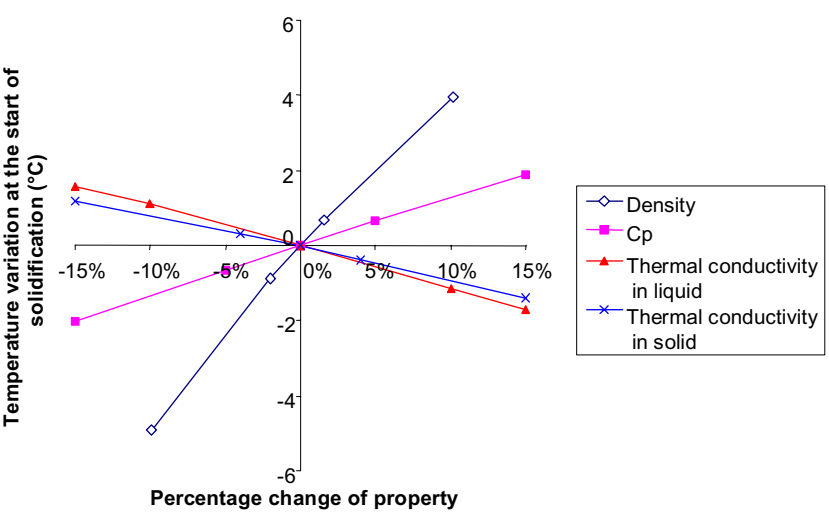

(a)

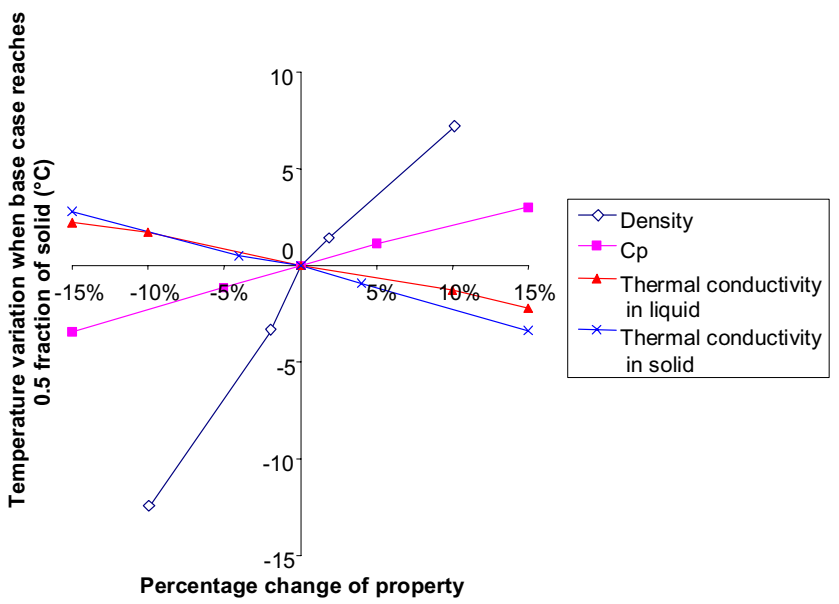

(b)

Fig. 2. The sensitivity of thermophysical properties on temperature variation in the mushy zone. (a) at the starting of solidification and (b) in the mushy zone with 0.5 fraction solid.

amount of latent heat released associated with the change of density.

Specific heat has a similar effect to density but its influence is less significant. Positive variation of $15 \%$ increases the temperature variation by $2{ }^{\circ} \mathrm{C}$ at the liquidus and $3{ }^{\circ} \mathrm{C}$ at 0.5 fraction solid.

Thermal conductivity has an inverse effect on the temperature variation: increasing its value decreases the temperature difference. The effect of thermal conductivity in the solid is slightly larger than that in the liquid. A positive $15 \%$ change of thermal conductivity in the solid decreases the temperature by $2{ }^{\circ} \mathrm{C}$ at the liquidus and $4{ }^{\circ} \mathrm{C}$ at 0.5 fraction solid.

Although from the form of the governing differential equations (Eq. (2)), one would expect similar magnitudes of responses for all variables, interestingly this is not the case. The temperature predictions show greatest sensitivity to density. This is due to the strong influence of the volumetric latent heat release term, of which the density is a pre-multiplier. Hence density influences the results both through altering this source term and through its impact on the rate of change of $T$ with time.

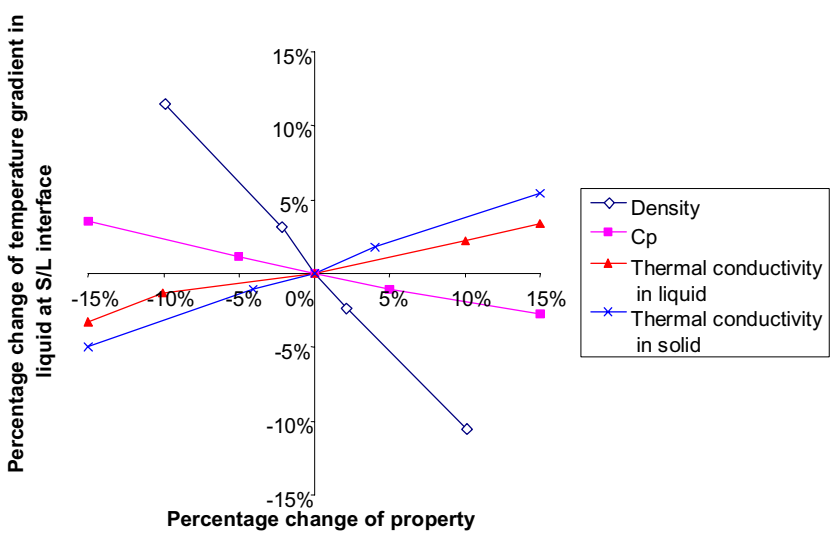

Fig. 3. The sensitivity of temperature gradient in liquid at $\mathrm{S} / \mathrm{L}$ interface to variations in the thermophysical properties.

$\underline{\text { Thermal Gradient in Liquid at Solid/liquid Interface }}$

The sensitivity of temperature gradient in the liquid at S/L interface to changes of the thermophysical properties is shown in Fig. 3.

An increase in thermal conductivity increases $G_{\mathrm{L}}$. However, the level of its influence is different in the solid and the liquid. Thermal conductivity in the solid shows a larger effect than in the liquid. A $15 \%$ increase in the solid's thermal conductivity increases $G_{\mathrm{L}}$ by $5.5 \%$ while the same variation of thermal conductivity in the liquid only increases $G_{\mathrm{L}}$ by $3.5 \%$. This is because the heat is conducted primarily through the solid state. Therefore, increasing thermal conductivity in the solid increases the heat conductivity rate more significantly, while increasing its value in the liquid increases the rate of heat release in the liquid which led to a more even temperature profile.

Density and $C_{\mathrm{P}}$ have inverse effects on $G_{\mathrm{L}}$ : increasing their values decreases the temperature gradient. This is due to an increased amount of heat accumulating in front of the interface. However, the effect of density is much larger than that of $C_{\mathrm{P}}$ since density is also associated with the volumetric latent heat release. Increasing its value by $10 \%$ decreases $\mathrm{G}_{\mathrm{L}}$ by $11 \%$ while increasing $C_{\mathrm{P}}$ by $15 \%$ only decreases $G_{\mathrm{L}}$ by $2 \%$.

The temperature gradient predictions show greatest sensitivity to density and thermal conductivity in solid.

\section{Local Solidification Time}

The sensitivity of local solidification time, $t_{\mathrm{s}}$, to changes in the thermophysical properties is shown in Fig. 4.

Density and specific heat have similar effect, increasing their values increases the local solidification time. This is due to an increase in the amount of heat that must be removed. But the effect of density is more significant than $C_{\mathrm{p}}$. A positive $10 \%$ change in density increases $t_{\mathrm{s}}$ by $3.2 \%$ while a $15 \%$ increase in $C_{\mathrm{p}}$ only increases $t_{\mathrm{s}}$ by $1.1 \%$. This can be explained from Eq. (2), where, density is also involved in the amount of latent heat to be removed. 


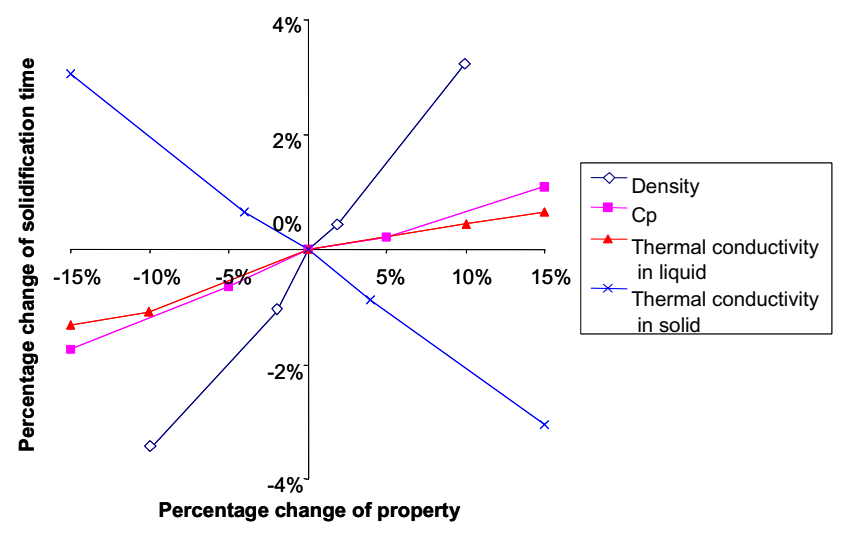

Fig. 4. The sensitivity of local solidification time to variations in the thermophysical properties.

Thermal conductivity in the liquid has a similar effect to density and $C_{\mathrm{p}}$ : increasing its value increases the local solidification time because more sensible heat is conducted to the semi-solid region from the liquid. Thermal conductivity in the solid has the inverse effect since an increase in its value will increase the rate of heat removal.

Local solidification time can be directly related to the secondary dendrite arm spacing, and hence influences the level of microsegregation, and is therefore one of the important outputs of casting simulations. However, large variations $(>10 \%)$ in the thermophysical properties produced less then a $4 \%$ change in the predicted $t_{\mathrm{S}}$

\section{Mesoscale Model Sensitivity}

The sensitivity of the microstructure to the accuracy in thermophysical data is investigated in this section by the simulating the primary dendrite arm spacing and microsegregation.

\section{$\underline{\text { Primary Dendrite Arm Spacing }}$}

Primary dendrite arm spacing is an important characteristic of columnar dendrites and has a marked effect on the mechanical properties [15]. In this section, the dependence of primary dendrite arm spacing to changes of the thermophysical properties is investigated.

A small area $\left(3 \times 10 \mathrm{~mm}^{2}\right)$ in the blade body was chosen as the simulation domain where the solidification morphology is dominated by growth of columnar dendrites. The location of the domain is shown in Fig. 1c at section I-I (hatched area) with initial conditions of one seed placed in the bottom corner of the domain.

Fig. 5 shows the typical dendritic microstructures predicted by the macro-meso model calculation where the density is changed by $\pm 10 \%$, together with the base case. Only a small part of the domain is shown in this figure in order to give a clearer representation of the dendritic structure. This simulation shows primary arm spacing increases with density. Average primary dendrite arm spacing is $130 \mu \mathrm{m}$ when density was changed by a negative $10 \%$ (Fig. 5a), and it increases to $158 \mu \mathrm{m}$ as density

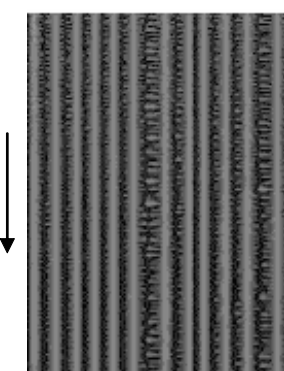

(a)
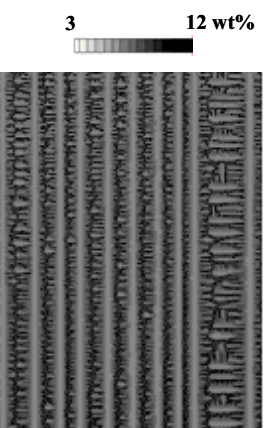

(b)

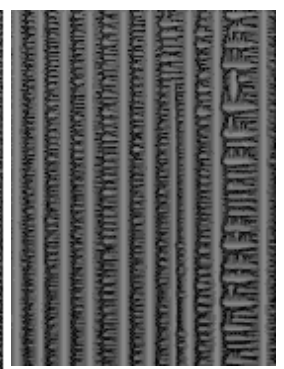

(c)
Fig. 5. Solidification microstructure. (a) density decreases by $10 \%$; (b) base case; (c) density increases by $10 \%$. Note: the arrow indicates withdrawal direction.

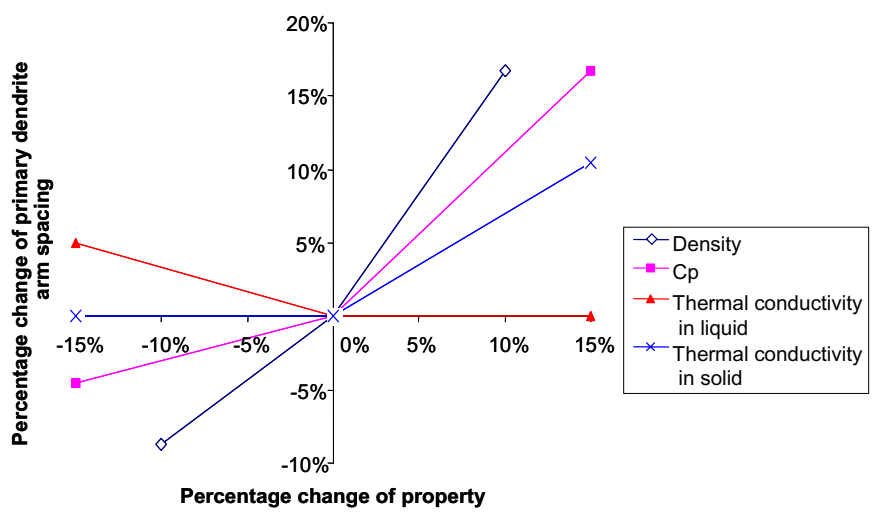

Fig. 6. Sensitivity of primary dendrite arm spacing to changes of thermophysical properties.

increased by a positive 10\% (Fig. 5c) whilst for the base case (Fig. $5 b)$ it is between the two extremes $(143 \mu \mathrm{m})$.

This simulation shows primary dendrite arm spacing increases with density. Measurements of the dendrite arm spacing as a function of the solidification conditions can be directly related to the tip velocity and the gradient ahead of the tips. These thermal properties can in turn be related to the local solidification time. As discussed in the last section, the solidification time shows greatest sensitivity to density. Therefore, as can be expected, primary dendrite arm spacing also shows the greatest sensitivity to density.

The percentage change in average primary dendrite arm spacing is shown in Fig. 6. Density and $C_{\mathrm{p}}$ have similar effects on the primary dendrite arm spacing, increasing their values increases the spacing. The effect of thermal conductivity on spacing is different in the liquid and in the solid: a negative change of thermal conductivity in the liquid by $15 \%$ increases the spacing by approximately $15 \%$ while a positive change of its value does not show much effect. Thermal conductivity in the solid has the opposite effect to that in the liquid: increasing its value by $15 \%$ increases the spacing by $10.5 \%$ whilst decreasing its value by $15 \%$ shows no change in the average spacing. This is due to the primary arm spacing being stable within a certain range [11, 15], although either a positive change of thermal conductivity in the liquid or a negative change of its value in the solid by $15 \%$ should decrease spacing, the primary arm spacing is still retained within a stable range. In addition, primary arm spacing is also dependent 


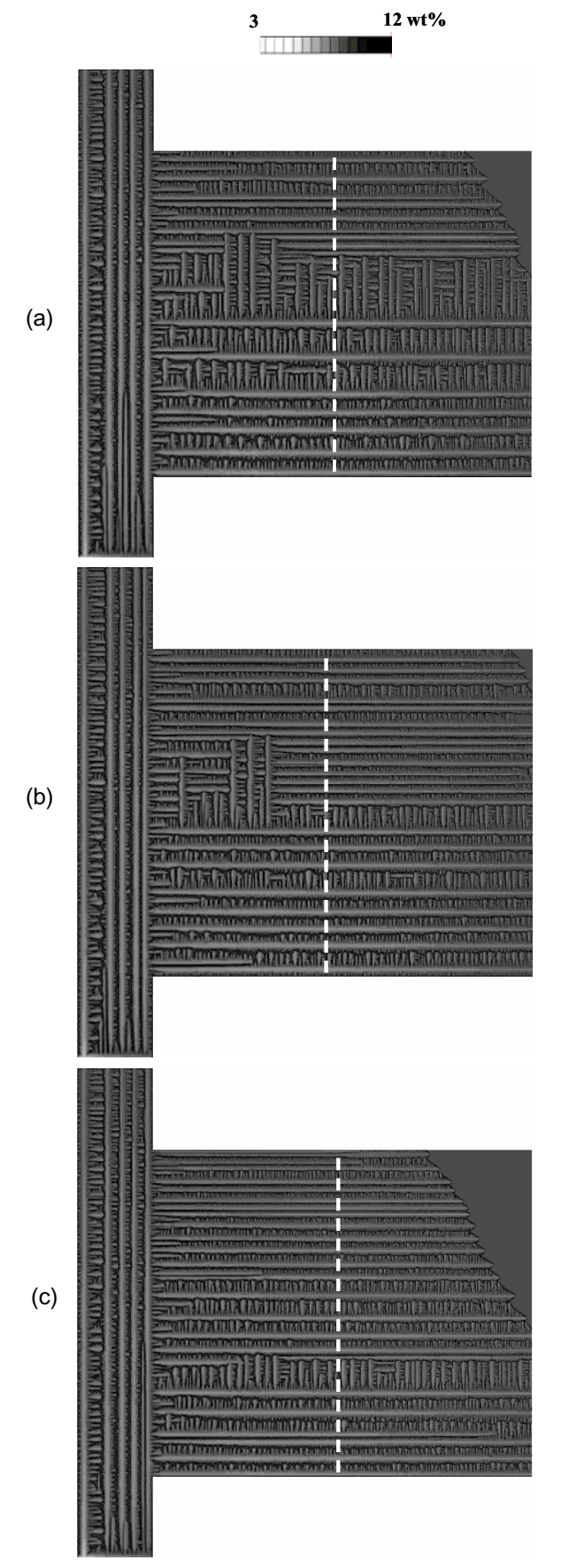

Fig. 7. Dendritic microstructure at inner platform. (a) density decreases by $10 \%$; (b) base case; (c) density increases by $10 \%$. Dashed line indicates the location of sampling line.

on the sequence of previous solidification conditions to which the system has been subjected. Therefore, there was no apparent change of primary spacing when some of the parameters were changed.

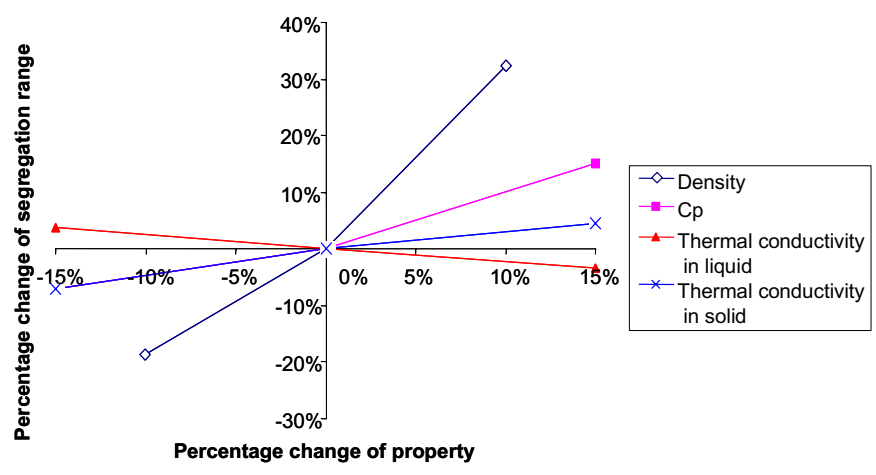

Fig. 8. Sensitivity of segregation range to changes of thermophysical properties.

Segregation Range

Sensitivity of the segregation range of the solidification microstructure to changes of thermophysical properties is examined here.

Fig. 7 shows the predicted microstructure in the inner platform of the blade for the base case and a change of $\pm 10 \%$ in density (Note: this zoomed simulation domain is shown in Fig. 1c of section II-II (hatched area).) As density decreases, the primary dendrites tend to form more secondary and tertiary dendrites, therefore, more complicated microstructures are produced. The influence of minor changes in the thermophysical property of density is magnified in this region, as compared to the core of the blade (see Fig. 5), since the chance of secondaries blocking primaries is heightened. By comparing Fig. 7 (a) to $7(\mathrm{c})$, the change from $-10 \%$ to $+10 \%$ variation in $\rho$ has a huge qualitative impact on the structures produced, much more so then when the geometry is simple (i.e. in the core of the blade).

To quantify this affect, a composition analysis was conducted for each case to obtain the concentration variation. One line (marked as a dashed line in Fig. 7) with $15 \mu \mathrm{m}$ grid spacing was extracted from the simulation results.

The level of microsegregation can be illustrated by the range of solute content. Here it is indicated by the difference between its maximum and minimum values in the extracted data. The segregation range was then calculated. The percentage change of the range is shown in Fig. 8.

The microsegregation range shows the greatest sensitivity to density, a positive $10 \%$ change of density increases the segregation range by $32 \%$ whilst a negative $10 \%$ change decreases the range by $18 \%$. Specific heat and thermal conductivity in the solid has a similar influence but the magnitude is reduced. Thermal conductivity in the liquid has the opposite effect to the other properties; increasing its value decreases the segregation range.

In order to determine the effect of changing thermophysical properties on the segregation path, five line scans were also performed with a total of 3650 points extracted for each of the conditions with changing of thermophysical properties. The locations of the 5 vertical lines are illustrated in Fig. 9. The average solute concentration was obtained along the vertical 


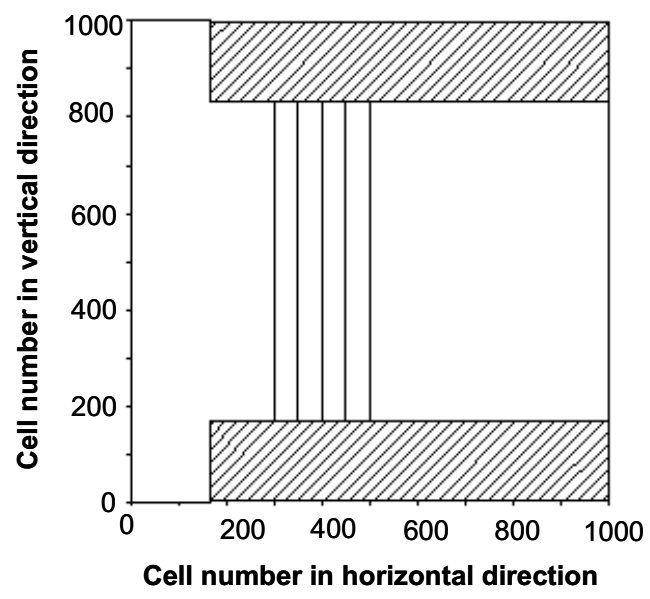

Fig. 9. Schematic illustration of simulation domain (inner side of platform) with 5 overlaid vertical sampling lines. Note: hatched area indicates mold wall.

direction. The resulting data sets were ordered and plotted against solid fraction. The data collected, ranked in an ascending order, are shown in Fig. 10. The concentration in solid is low at the start of solidification. It increases as the solidification process continues. As the solidification process approaches its end, the concentration increases and approaches its maximum.

The effect of changing thermophysical properties on the extent of segregation is shown by the different curves in Fig. 10. Increasing thermal conductivity increases the segregation level but with less magnitude in the liquid. Specific heat, $C_{\mathrm{P}}$, has an inverse effect; increasing its value decreases the segregation. Density has a similar effect to thermal conductivity; increasing its values increases the segregation.

The greater the dendrite arm spacing and the higher the segregation range the more difficult is homogenization by subsequent heat treatment [16]. From this investigation, density and solid state thermal conductivity were found to strongly effect both dendrite arm spacing and the microsegregation, therefore, an accurate measurement of these values is important.

\section{Conclusions}

The sensitivity of investment casting simulations to the accuracy of thermophysical properties was investigated in terms of thermal field and solidification microstructure. The sensitivity of temperature distribution, thermal gradient in the liquid at $\mathrm{S} / \mathrm{L}$ interface, and local solidification time were examined using a macroscale ProCAST model. The temperature prediction and thermal gradient in the liquid are the most sensitive to the accuracy of the values used for density and thermal conductivity in the solid. Thermal conductivity in the liquid, specific heat and density have similar levels of influence on solidification time; increasing their values increases the local solidification. Thermal conductivity in the solid has the opposite effect compared to all the other properties studied. However, the magnitude of this change is small. Altering the conductivity by $15 \%$ only changes the solidification time by $4 \%$. The responses of primary dendrite arm spacing and segregation range was investigated using a

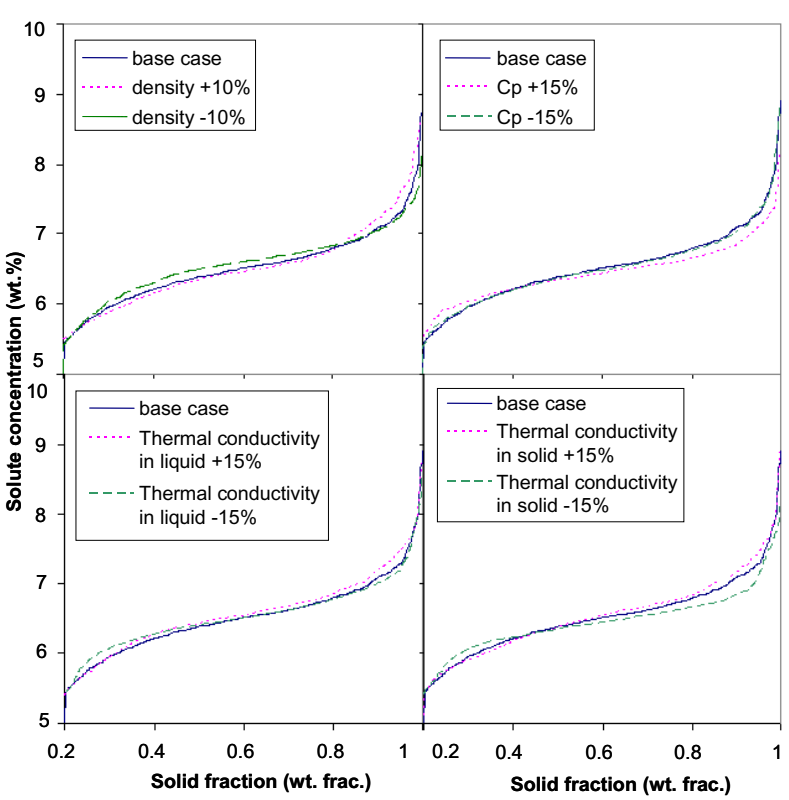

Fig. 10. Microsegregation profile for the solute under the change of thermophysical property dada.

coupled macro-meso scale model. Primary arm spacing shows greatest sensitivity to density and $C_{\mathrm{p}}$. The extent of microsegregation showed the greatest sensitivity to density and thermal diffusivity in the solid.

In summary, accurate thermophysical property data are required for accurate predictions, with meso-models being more sensitivity in many cases than macromodels to variations in the data.

\section{Acknowledgements}

The authors would like to thank the EPSRC (GR/R78992) and Rolls-Royce plc for financial support together with the provision of thermal model and experimental information. The authors would also like to thank ESI Software, Paris, for the provision of the ProCAST software. The assistance of Dr. H. B. Dong and other colleagues in the Materials Processing Group, Imperial College, is gratefully acknowledged.

\section{References}

1. K.C. Mills et al., "Some cautionary notes on the measurement and use of thermophysical property data," High Temp.-High Press., 34 (2002), 252-264.

2. K.C. Mills and P.D. Lee, "Thermophysical property requirements for modeling of high temperature processes," (paper presented at the First International Symposium on Microgravity Research \& Applications in Physical Sciences and Biotechnology, Sorrento, Italy, 2001), 555.

3. G. Höhne, W. Hemminger, and H-J. Flammersheim, Differential scanning calorimetry an introduction for practitioners (Springer -Verlag Berlin and Heidelberg GmbH \& Co., 1995). 
4. T. Iida and R I.L. Gurthrie, The physical properties of liquid metals (Oxford, Clarendon Press, 1988).

5. L. Dusza, "Combined solution of the simultaneous heat loss and finite pulse corrections with the laser flash method," High temp. - High. press., 27 (1995), 467-471.

6. R.B. Bird, W.E. Stewart, and E.N. Lightfoot, Transport phenomena (Singapore, John Wiley \& Sons, Inc., 1960), 318.

7. "ProCAST User's Manual \& Technical Reference" (UES Software, Inc., 1998), C-10.

8. P. Carter et al., "Process modeling of grain selection during the solidification of single crystal superalloy castings," Mater. Sci. Eng. A, 280 (2) (2000), 233-246.

9. P.A. Jennings, "Foundries Satellite VeriCAST. Phase I Simple Blade Casting Trials and Thermal History GenerationFinal Report" (Report CRDF/R96/1, Rolls-Royce plc., 1996).

10. P.D. Lee et al., "Modeling of porosity formation in direct chill cast aluminum- magnesium alloys," Mater. Sci. Eng. A, 328 (2002) (1-2), 213-222.

11. W. Wang, P.D. Lee, and M. McLean, "A model of solidification microstructures in nickel based superalloys: predicting primary dendrite spacing," Acta Mater., 51 (10) (2003), 2971-2987.

12. C.-A. Gandin and M. Rappaz, "A 3D cellular automaton algorithm for the prediction of dendritic grain growth," Acta Mater., 45 (5) (1997), 2187-2195.

13. X.L. Yang et al., "Microscale simulation of stray grain formation in investment cast turbine blades," Mater. Sci. Eng. A, (2004), submitted.

14. K.C. Mills, Recommened values of thermophysical properties for selected commercial alloys (Woodhead publishing Ltd and ASM international, 2002), 166-174.

15. J. D. Hunt and S.Z. Lu, "Numerical modeling of cellular dendritic array growth: Spacing and structure predictions," Metal. Mater. Trans. A, 27 (3) (1996), 611-623.

16. G.J. Davies, Solidification and casting (Barking, Applied Science Publishers Ltd., 1973), 58. 